\title{
The CMS RPC system - detector performance and upgrade
}

\section{Min Suk Kim* On behalf of the CMS Collaboration}

Sungkyunkwan University, Department of Physics, 2066 Seoburo, Jangangu, Suwon, Korea (Now at Kyungpook National University, Department of Physics, 80 Daehakro, Bukgu, Daegu)

E-mail: minsukecern.ch

The Resistive Plate Chambers are used in CMS as dedicated muon trigger detector both in the barrel and in the two endcap regions. They also contribute to the identification of the muons, together with Drift Tube in the barrel and Cathode Strip Chambers in the endcaps. About 4000 square meter of double gap RPCs have been produced and have been installed in the experiment since 2007. We will present the performance study and operational experience after three years of successful data taking at LHC. The upgrade program foreseen for the next two years also will be outlined.

The European Physical Society Conference on High Energy Physics -EPS-HEP2013

18-24 July 2013

Stockholm, Sweden

\footnotetext{
${ }^{*}$ Speaker.
} 


\section{Introduction}

The Resistive Plate Chambers (RPCs) system for the Compact Muon Solenoid (CMS) experiment is one of the largest systems based on RPCs ever built. RPCs are gaseous detector for ionizing particles, and have been fulfilling performance needs in muon triggering and identification for the first three-year running period of data taking at the LHC at CERN near Geneva, Switzerland.

The RPC chambers are very important detectors because of excellent time resolution which is crucial at the LHC due to a $25 \mathrm{~ns}$ spacing between proton bunches in the beams. They are thus used as dedicated fast trigger detectors and complement the triggering capabilities of the Drift Tubes (DTs) and Cathode Strip Chambers (CSCs) in the CMS muon system. The good performance of RPCs is very essential in assigning the muon to the right bunch crossing. In addition to serving as dedicated triggers, the RPC system also contributes the muon reconstruction [1].

\section{The CMS RPC system}

The RPCs used in CMS are bakelite-based, double-gap detectors with bulk resistivity ranges $\sim 10^{10} \Omega \cdot \mathrm{cm}$. Each gap is composed by two parallel electrodes, each of $2 \mathrm{~mm}$ thickness, and a $2 \mathrm{~mm}$ wide gas gap, and operated with a gas mixture in avalanche mode to safeguard the time resolution at high rates $\left(\sim 1 \mathrm{kHz} / \mathrm{cm}^{2}\right)$. The water vapor is added to keep its relative humidity around $45 \%$ so that the bakelite resistivity remains constant, avoiding a degradation of performance under high background conditions. Readout strips along the beam direction are sandwiched between two gaps, with an independent electronic channel per strip, segmented in pseudo-rapidity $\eta$.

Figure 1 shows muon stations that are placed in the steel return yoke of the magnet (gray). The four DT stations in the barrel (yellow), the four CSC stations in the endcap (green), and the RPCs in both barrel and endcap (blue) are shown. During the first three-year LHC running period, the 4 th endcap stations remained largely uninstrumented. CSCs were only installed in the very forward region $(1.8<|\eta|<2.4)$, and RPCs were missing completely and covered only the first three endcap disks $(|\eta|<1.6)$. During the first long shutdown (LS1) in 2013-2014, these 4th endcap stations will be instrumented further with new CSCs (ME4) and RPCs (RE4) up to $|\eta|=1.8$. An average efficiency increase by both ME4 and RE4 was found to be $\sim 2 \%$ for $1.2<|\eta|<1.8$ in the simulation.
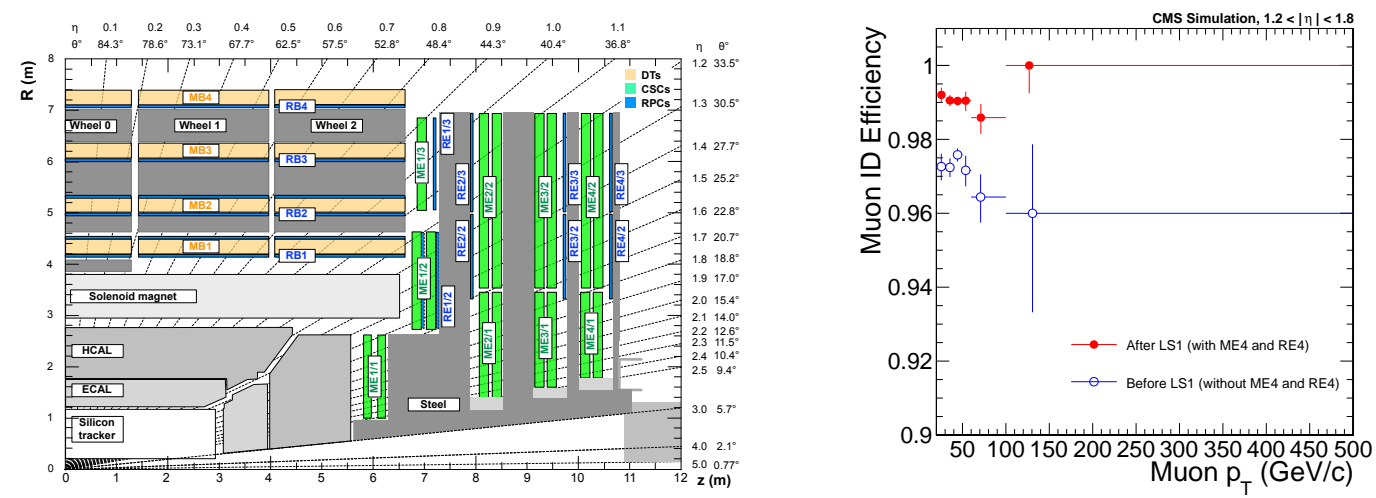

Figure 1: Layout of one quadrant of the CMS detector (left) and efficiencies for prompt muons by applying a tag-and-probe technique to muons from $\mathrm{Z}$ decays as a function of $p_{T}$ before and after LS1 (right). 


\section{The Calibration of the system}

When a particle ionizes the gas between the electrodes, an avalanche process occurs. This process depends on the environmental conditions, in particular pressure and temperature, directly affecting gas density and so on. Thus, one of the main reasons of possible performance variations in the RPCs is the applied High Voltage (HV) with the gas-mixture fixed.

To optimize the performance at the single chamber level, data of a series of dedicated runs (called HV Scans) at eleven HV settings from 8.5 to $9.7 \mathrm{kV}$ in 2011-2012 have been studied. The chamber efficiency was determined by extrapolating the muon tracks spotted in the nearby detectors (DTs and/or CSCs) and finding the RPC hits in a certain fiducial region [2]. The optimal operating voltage for each chamber has been determined using the standard procedure. We observed that the efficiency measured in the HV Scans has not been varying significantly in two years.

Figure 2 shows that the applied calibration succeeded in keeping the average efficiency ( $95 \%)$ stable within $\approx 2-3 \%$ fluctuations by requiring the gas density to remain constant. This means that the performance of the RPC system for muon tracking and trigger is stable in time, which is one of the greatest accomplishments in its essential functions during the 2012 data taking period.

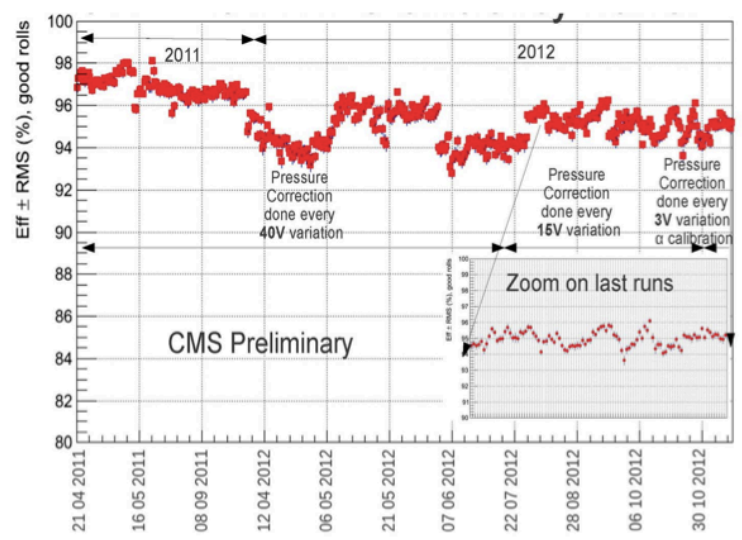

Figure 2: Average RPC barrel efficiency, starting from April 2011 until late 2012. A zoom of the last period shows more refined algorithms applied for the calibration of the system.

\section{Conclusions}

Using enough statistics collected during 2011-2012 with increasing instantaneous luminosity, the calibration of the RPC system has been developed to improve data quality for physics. As the LHC begun its first long shutdown, the system is undergoing its foreseen maintenance, and a fourth disk in both endcaps is being installed, making the system more robust and efficient than before.

\section{References}

[1] M. S. Kim, CMS reconstruction improvement for the muon tracking by the RPC chambers, JINST 8 (2013) T03001.

[2] S. Costantini et al., Uniformity and Stability of the CMS RPC Detector at the LHC, JINST 8 (2013) P03017. 